\title{
Education
}

\section{An Obstetrics and Gynecology Residency Program's Approach to the COVID-19 Pandemic}

Tiffany C. Chang, MD, Brenda Nguyen, MD,' Chi Dola, MD, MPH'1

\section{Abstract}

Author affiliations are listed at the end of this article.

Correspondence to: Chi Dola, MD, MPH 1430 Tulane Ave, Box 8611 New Orleans, LA 70112 (cdola@tulane.edu)

\section{Background}

The impact of COVID-19 on residency training nationwide has been substantial, and adapting to this unprecedented event has proven challenging for program directors throughout the United States. Here, the authors presented their initial experiences with restructuring an obstetrics and gynecology residency program during the pandemic. The authors outlined their strategies to maximize resident safety and address clinical care in outpatient and inpatient settings, resident education curriculum, resident wellness and consider the ethical dilemmas of health care providers during a pandemic.

\section{Conclusion}

With perspectives from other residency programs, the authors hope this review will serve as an initial building block for developing an effective strategic response for programs to implement during a disaster.

\section{Keywords}

COVID-19; SARS-CoV-2; coronavirus infections; pandemics; preparatory response; internships and residency; graduate medical education; occupational health; obstetrics; gynecology; distance education; telemedicine; disease outbreaks/prevention \& control

\section{Introduction}

On March 11, 2020, the World Health Organization declared a global pandemic from the novel severe acute respiratory syndrome-coronavirus 2 (SARS-CoV-2), recognized to cause coronavirus disease 2019 (COVID-19). ${ }^{1}$ Rapid spread of the pandemic in the United States has severely disrupted the healthcare system, posing unprecedented challenges to residency training programs. Specifically, recent characterization of its highly infectious nature and potential for asymptomatic transmission 2,3 have guided infection control recommendations to minimize exposure, adhere to safety precautions and reduce to essential activities. ${ }^{4}$ Due to the demands of resource allocation and social distancing, elective surgeries and clinic visits were cancelled or postponed, severely impacting most surgical subspecialties and residency training programs.
However, in obstetrics, pregnancy care must continue and birth simply cannot be postponed. Therefore, obstetrics and gynecology residency programs must quickly strategize to provide adequate patient care and avoid illness in health care providers. Current recommendations for disaster preparedness are directed towards hospital administration rather than residency-specific operations. ${ }^{5}$ Early in the pandemic, program directors across the country quickly employed group email communication chains to propose and discuss potential plans for redesigning programs. Currently, available data evaluating recent restructuring strategies are limited. ${ }^{6,7}$ Therefore, we outlined our program's early responses to maximize resident safety and effectively care for the obstetrics population in the face of a COVID-19 public health emergency.

\section{HCA 位 Healthcare}

\section{www.hcahealthcarejournal.com}

(C) 2020 HCA Physician Services, Inc. d/b/a Emerald Medical Education
HCA Healthcare Journal of Medicine 


\section{General Program Redesign}

Our previous team structure consisted of 4 teams of residents covering 4 major sites. During the pandemic, this resident workforce was restructured into two new teams based on the level of patient contact: Team 1 participated in clinical duties with direct patient contact and Team 2 was responsible for activities without direct patient contact, rotating every 14 days. This team structure safeguarded our entire residency cohort from simultaneous infection and a potential workforce shortage. Specifically, Team 1 covered all obstetrical services, emergent gynecological services and ambulatory clinics, for which in-person appointments were limited to prenatal care and emergent gynecological care. Team 2 sequestered at home to minimize exposure and served as back-up for clinical services with direct patient contact, should any resident become ill. Team 2 covered all telehealth and telephone visits from home and finalized any incomplete medical records. They also utilized educational resources provided for independent learning, allocated time for research projects and completed other scholarly activities, including quality improvement projects and residency presentations (e.g., grand rounds, subspecialty conferences). Accordingly, educational goals and objectives were revised to reflect the restructured clinical activities.

\section{Resident Wellness}

With health care providers adversely impacted by the unprecedented changes and uncertainty surrounding the pandemic, resident wellness and safety are an especially important priority during this time. When social distancing recommendations were issued, the program took necessary measures to prepare residents for the potential challenges posed by the COVID-19 pandemic, including the opportunity for at-risk residents to forgo clinical duties without impunity. Under an honor code, at-risk residents with underlying medical conditions could take a temporary leave from clinical duties conveying high risk of exposure and were assured of continued compensation and benefits established prior to the pandemic.

Regular residency program meetings on Mondays, Wednesdays and Fridays over teleconference Zoom software (Zoom Video Communications, San Jose, CA) were instituted to provide updates, support and open routes of communication for all residents, especially those sequestering at home. The Tulane School of Medicine Department of Psychiatry and Behavioral Services introduced no cost mental health services for residents and resources for telehealth counseling services with licensed clinical therapists. With the rapid spread of COVID-19, fears and anxiety were exacerbated by the shortage of personal protective equipment (PPE). Program leadership asked and received widespread support from medical students, who built face shields at low costs to combat the PPE shortage.

Safety concerns of essential workers juggling occupational and caregiver responsibilities were further complicated by local stay-athome orders requiring school closures. To address these concerns regarding childcare while at work, the medical school provided daily financial support of $\$ 100$ for childcare. For residents concerned about bringing SARSCoV-2 infections to household members from exposures at work, the university offered free local housing at a graduate housing facility or nearby hotels.

\section{Ethical Considerations}

During the widespread uncertainty of the pandemic, residents faced the unprecedented challenge of managing highly infectious patients. Residents were given the opportunity to express their justified fears and concerns. The ethical debate concerning health care workers' duty to treat, especially during pandemic, was discussed during morning rounds. ${ }^{8}$ Specifically, the American Medical Association's COVID-19 ethics guidance was reviewed and discussed amongst residents, who were reminded that physicians have an ethical obligation to provide urgent care to patients even under circumstances that may subject their own health, life or safety to increased risk. ${ }^{9}$ This duty is limited, however, by the potential risk of physician absence because of illness and the subsequent workforce shortage. ${ }^{9}$ It is therefore necessary that residents with underlying medical conditions be given the opportunity to "opt-out" with no questions asked.

When Tulane University was granted Stage 3: Pandemic Emergency Status by the Accredi- 
tation Council for Graduate Medical Education (ACGME), residents across all specialties were asked to redeploy to internal medicine wards and intensive care units to assist our colleagues with patient care. However, it was emphasized that clinical redeployment was entirely voluntary and not proscribed. Work hour limits continued to be strictly enforced to mitigate the risk for errors resulting in serious consequences, including contamination and infection exposure.

\section{Clinical Care}

Major efforts were directed towards developing clinical strategies to optimize patient care, while maintaining a physical distance. We limited exposure to potentially infectious individuals by postponing non-essential clinical visits and elective procedures.

Infection control practices were widely discussed with residents and the labor and delivery workflow was redesigned in accordance with recent practice guidelines for managing COVID-19 in pregnancy. ${ }^{10,11}$ Regular updates on the rapidly changing landscape of the pandemic were also discussed during daily multidisciplinary morning rounds. Challenges of the newly designed workflow were presented by those working in the trenches and collectively, solutions were devised.

\section{Designated COVID Team}

We constructed a COVID team to exclusively attend to confirmed COVID-19 patients or persons under investigation (PUI), limiting exposure to presumed healthy team members and patients. This team structure was based on principles driving the Resident Emergency Code Grey (in response to natural disasters) Team instituted by our program since Hurricane Katrina ravaged the Gulf Coast, greatly affecting local residency programs.

We imposed strictly voluntary participation of residents on the COVID team. Only senior residents (PGY-III and PGY-IV) without excess risk for critical COVID-19 illness or heavy family responsibility that could risk household transmission of infection could qualify for the team. Accounting for the American Board of Obstetricians and Gynecologists qualifying exam in June, fourth year residents participated on the COVID team in the early weeks of the pan- demic, while third year residents participated in the weeks leading up to the examination, to prevent transmission to fourth year residents nearing their examination.

The COVID team consisted of one upper level resident, led by either a Maternal-Fetal Medicine (MFM) attending or generalist attending with a MFM specialist as consultant. The COVID team took calls from home, rotating every 7 days. Their clinical duties included all triages, admissions, procedures, daily rounds and discharges for COVID-19 patients and PUI. The COVID team reviewed proper donning and doffing procedures daily. No other clinical duties were assigned to the resident on COVID team in order to mitigate competing tasks for the resident during a stressful time and inadvertently contaminate other patients and colleagues. However, the COVID team resident was expected to complete educational assignments during downtime and be an expert on COVID-19 infection.

Separate labor and delivery rooms, designated as the OB COVID unit, operating rooms and postpartum rooms were reserved for COVID patients. Inter-team isolation and distancing measures were also taken to identify workstations, call rooms, showers and changing rooms for use only by the COVID team, who showered and disposed of scrubs prior to returning home. We recommended twice-daily temperature checks and self-monitoring of COVID-19 symptoms for 14 days following contact with COVID-19 patients.

\section{Initial Screening and Testing for COVID-19}

In the beginning of the outbreak, residents quickly learned the practical use of the public health strategy to "flatten the curve." Residents were taught to make triage decisions based on reported symptoms of COVID-19. They postponed visits for patients with positive screening results who presented for non-urgent gynecological concerns or diagnostic procedures (e.g., screening mammograms) and referred these patients to drive-through testing centers.

Institutional access to one of the first commercial COVID-19 diagnostic polymerase chain reaction tests, Cobas ${ }^{\circledR}$ SARS-CoV-2 Test (Roche 
Molecular Systems Pleasanton, CA), combined with increasing reports of asymptomatic infection and transmission, ${ }^{3}$ led to our institution's universal testing of all patients $1-2$ days prior to scheduled procedures (e.g., induction, cesarean delivery and cerclage). With the availability of a newly authorized rapid point-of-care testing, ID NOW (Abbott Diagnostics, Scarborough, ME), all patients presenting for triage or admission were tested prior to entry to the labor unit. Patients testing positive were escorted directly to the negative pressure OB COVID unit. Patients with emergent concerns (e.g., advanced active labor) and pending test results were also escorted directly to the OB COVID unit. All patients' support persons were tested for COVID-19 infection, including alternative support persons if the former tested positive.

\section{Labor And Delivery Protocol}

Modified intrapartum care practices, based on recent guidelines for the management of labor during the COVID-19 pandemic, ${ }^{10}$ were discussed and the adjustments were adopted by residents on the labor and delivery unit. Common obstetric practices were modified, such as careful consideration of betamethasone therapy, safe early hospital discharge, blood pressure devices prescribed at discharge and thorough patient counseling for postpartum conditions that warrant telephone calls to providers. The decision to proceed with cesarean section for fetal non-reassuring status was made earlier to avoid emergent cesarean section, which could increase the risk of exposure for health care providers. Certain practices with low evidence-based recommendations were avoided, including elimination of the peanut ball, which is placed between the laboring woman's legs to purportedly facilitate descent of the fetal head during labor and also is a potential vector for infection. We also avoided certain procedures including non-medically indicated artificial rupture of membranes to prevent risk of umbilical cord prolapse, an obstetric emergency with high rates of fetal morbidity and mortality that indicate emergent delivery, typically through cesarean section.

Use of proper PPE with N95 masks and face shields, critical during the second stage of labor and during delivery, was implemented at all deliveries, in accordance with the Society of Maternal-Fetal Medicine (SMFM) and the
American College of Obstetricians and Gynecologists (ACOG) recommendations. ${ }^{12,13}$ For vaginal deliveries, we imposed an additional protective barrier by affixing the readily available abdominal drapes to IV poles on either sides of the bed..$^{4}$ Deliveries were limited to one resident and one attending, considering the need to conserve PPE and minimize unnecessary exposure.

\section{Consultation Service}

We adopted "curbside" services for inpatient and emergency department consultations for non-urgent complaints identified to not pose medical harm or change inpatient management plans. This allowed us to better allocate our resources (e.g., PPE) and time to patients with urgent needs while also minimizing unnecessary exposure.

\section{Outpatient Service \\ Prenatal care}

Guidance on prenatal care by Boelig et al. was available early in the pandemic and used to develop a modified prenatal care schedule for the resident-run clinic. ${ }^{11}$ In brief, in-person prenatal visits were interspersed with telephone evaluations or telehealth as clinically appropriate. Routine laboratory tests, ultrasounds and antenatal tests were completed at in-person prenatal visits. Residents received training to provide telephone evaluations and appropriate documentation for these visits. Residents prescribed blood pressure devices to all patients, who were instructed to take blood pressure and body weight measurements at specific time intervals for review during the telephone visits.

Ultrasonography exams were strategically scheduled to optimize visualization of fetal anatomy and decrease the need for a follow-up exam." Antenatal surveillance of fetal growth and non-stress testing were spaced out with careful evaluation of the risks to mother and fetus, as per Boelig et al. ${ }^{11}$ This modified surveillance schedule for lower risk pregnancies ultimately did not alter the course of obstetrical management, but rather, reduced burden on patients to attend frequent routine prenatal visits during the COVID-19 pandemic. The successfully modified prenatal schedule for lower risk pregnancy may thus, serve as a potential 
nidus for change in clinical behavior moving forward.

Residents performed postpartum follow-up telephone evaluation within one week of early hospital discharge. Additional follow-up appointments, including telephone or in-person care, were provided as indicated. For patients with an uncomplicated postpartum period, the in-person postpartum care visit could therefore be delayed up to 8 weeks after delivery.

\section{Outpatient Gynecology Clinic And Subspecialty Care}

Residents reviewed clinic schedules, for which non-urgent gynecological and subspecialty clinic visits were postponed or transitioned to telehealth sessions, reducing in-person appointments to urgent concerns or necessary gynecologic procedures. These virtual visits were scheduled to prevent feelings of provider abandonment and provide reassurance to patients. To minimize exposure risk for patients and clinical personnel, all patients were advised by our residents to seek telehealth services for first line clinical guidance instead of visiting the emergency department.

Non-patient-contact learning opportunities were also created for residents. Through the Maternal-Fetal Medicine clinics, residents studied fetal anatomy and fetal biometry by remotely reviewing images obtained by technicians under the guidance of attending physicians. In addition, residents read mammograms and breast ultrasound examinations remotely with the general surgery attending covering the breast clinic.

\section{Resident Education Didactic education}

Weekly teaching conferences have been conducted virtually to preserve resident engagement and active learning. With restrictions on large in-person gatherings, we adopted a teleconference format to ensure continuation of important didactic education. Early in the pandemic, resident didactics consisted of MFM attending-led discussions of the most recent literature and management of COVID-19 infection in pregnancy. Updated resources from governing organizations, including SMFM, ACOG and the Society for Obstetric Anesthe- sia and Perinatology established the fundamental educational curriculum for our residents during the rapidly evolving pandemic. ${ }^{13-15}$ Early resident didactic education was supplemented by basics of critical care lectures and reviews of cardiopulmonary resuscitation and acute respiratory failure; and later, distance learning through the National Remote Obstetrics and Gynecology Resident Didactics, from some of the best academic faculty across the country, was included. ${ }^{16}$

Residents demonstrated critical thinking skills by discussing new research findings and applying these new developments to the evolving checklist and management algorithm. Through these virtual meetings, residents on reserve stayed informed about new insights and personal experiences from colleagues to ultimately prepare for return to in-person clinical practice.

\section{Individualized Independent Learning Curriculum}

Keeping up with current literature and academics is essential for any obstetrics and gynecology residency program. Residents on reserve had the unique opportunity to develop an individualized learning curriculum with goal-oriented weekly assignments reviewed by faculty and distance learning resources. Residents received immediate feedback from quizzes after each reading assignment. Quizzes on the assigned reading material were submitted weekly and the total number of correct answers for each resident were tabulated and updated in real-time to the program director's dashboard. These educational materials included contracted Decker's Weekly Curriculum, TrueLearn SmartBanks and American Society for Colposcopy and Cervical Pathology (ASCCP) modules. ACOG's Surgical and Fundamentals of Laparoscopy modules were always available to residents. During the pandemic, residents were also generously provided complementary access to other scientific websites (e.g., SAGE, SGO, IAPS, Rosh Review) for additional educational material.

Off-site education served as an opportunity for residents to further explore scholarly interests, including publishing peer review reports, data analysis, retrospective research and case reports describing early experiences with COVID-19 in pregnancy. The overwhelm- 
ing scholarly response from residents appears somewhat cathartic, as an outlet for residents to sort through their experiences of being an obstetrics and gynecology resident during a global pandemic emergency.

\section{Outlook And Conclusions}

In considering the experiences and challenges posed in the COVID-19 pandemic, we have had the opportunity to reflect on our ethical duties as physicians and our responsibilities to reevaluate the cost of care (e.g., educating our colleagues on optimal use of consultation services) and to allocate scarce resources. Residents have learned, through firsthand experiences, the foreign concept of social distancing, an intervention widely used in public health to combat the spread of infectious disease. Instead of traditional patient-centered care, residents learned to adapt modified obstetrical practices that benefit the community at large. ${ }^{17}$ Residents have also had greater opportunity to master systems-based practices, one of the ACGME Core Competencies. Through frequent changes to hospital policies and algorithms during the rapidly changing landscape of the pandemic, residents learned to quickly adapt to numerous healthcare systems and protocols at various hospitals.

The restructuring of our residency program has captured unexpected resident education benefits of valuable experiences previously difficult to integrate into clinical care-heavy schedules, including focused ethical discussions, implementing action plans on the public health response to COVID-19, scholarly activities and developing systems-based practice skills in the face of changing hospital guidelines and protocols, resource rationing and individvalized learning. Without the opportunity to restructure the program, albeit under unfortunate circumstances, remote learning resources, including individualized self-learning and videoconferencing, may have never been explored as an alternative to didactic conference. We have benefited from weekly virtual conferences of topics presented by some of the best academicians in the country and hope these available resources continue beyond the pandemic. Amidst these constant changes in a tumultuous pandemic period, our university and the state of Lovisiana formed a united front to overcome initial hurdles, from which valuable lessons could not have been learned through routine clinical care or effectively taught through didactics. Before the outbreak, our patient-centered clinical practice emphasized family/friend involvement, continuity of care with close follow-up and in-person support with minimal consideration of electronic or virtual modalities. However, our focus has quickly shifted to a community-centered practice advocating for public health to protect the community at large and reduce the burden of continued COVID-19 transmission on the healthcare system. Social distancing measures and "flattening the curve," previously not topics of general knowledge, are now widely discussed in order to improve public health, ration resources and reduce general overload of the healthcare system. Health care personnel at the frontlines continue facing unprecedented change and uncertainty, as researchers race to elucidate a multifaceted understanding of this novel viral illness. The fluidity of this public health emergency has posed a challenge for residency programs to quickly and effectively restructure the resident workforce.

There are limited published data about preparing an obstetrics and gynecology residency training program, highlighting the need for combining strategies to formulate a solution. We describe our early experiences in an effort to identify strategies that best address the challenges posed by the pandemic uncertainty. Through shared strategies and perspectives of other obstetrics and gynecology residency programs, we hope to initiate a collaborative effort to outline the most effective solution for programs to implement during any disaster response. In addition, our experiences can potentially provide the framework for future research on modified obstetric practices that alleviate the burden on patients without increasing risk to the mother or fetus (e.g., decreasing the number of prenatal visits and the frequency of antenatal surveillance) by comparing outcomes to historical norms and ultimately, create new protocols for improved patient care.

\section{Conflicts of Interest}

The authors declare they have no conflicts of interest.

The authors are employees of Tulane University School of Medicine with practices at Tulane- 
Lakeside Hospital, a hospital affiliated with the journal's publisher.

This research was supported (in whole or in part) by HCA Healthcare and/or an HCA Healthcare affiliated entity. The views expressed in this publication represent those of the author(s) and do not necessarily represent the official views of HCA Healthcare or any of its affiliated entities.

\section{Author Affiliations}

1. Tulane University School of Medicine, New Orleans, LA

\section{References}

1. World Health Organization. WHO Timeline COVID-19. https://www.who.int/news-room/ detail/27-04-2020-who-timeline---covid-19. Published 2020. Updated April 27, 2020. Accessed May 1, 2020.

2. van Doremalen N, Bushmaker T, Morris DH, et al. Aerosol and Surface Stability of SARS-CoV-2 as Compared with SARS-CoV-1. N Engl J Med. 2020;382(16):1564-1567. https://doi.org/10.1056/ nejmc2004973

3. Bai Y, Yao L, Wei T, et al. Presumed Asymptomatic Carrier Transmission of COVID-19. JAMA. 2020;323(14):1406-1407. https://doi.org/10.1001/ jama.2020.2565

4. Centers for Disease Control and Prevention. Interim Infection Prevention and Control Recommendations for Patients with Suspected or Confirmed Coronavirus Disease 2019 (COVID-19) in Healthcare Settings. https://www.cdc.gov/coronavirus/2019-ncov/hcp/infection-control-recommendations.html. Published 2020. Updated Apr 13. Accessed Apr 19, 2020.

5. Committee on Obstetric Practice and the American Academy of Pediatrics' Council on Environmental Health. Committee Opinion No. 726: Hospital Disaster Preparedness for Obstetricians and Facilities Providing Maternity Care. Obstet Gynecol. 2017;130(6):e291-e297. https:// doi.org/10.1097/aog.0000000000002413

6. Schwartz AM, Wilson JM, Boden SD, Moore TJ Jr, Bradbury TL Jr, Fletcher ND. Managing Resident Workforce and Education During the COVID-19 Pandemic: Evolving Strategies and Lessons Learned. JB JS Open Access. 2020;5(2):e0045. Published 2020 Apr 15. https:// doi.org/10.2106/jbjs.oa.20.00045

7. Vargo E, Ali M, Henry F, et al. Cleveland Clinic Akron General Urology Residency Program's COVID-19 Experience. Urology. 2020;140:1-3. https://doi.org/10.1016/j.urology.2020.04.001

8. Malm H, May T, Francis LP, Omer SB, Salmon DA, Hood R. Ethics, pandemics, and the duty to treat. Am J Bioeth. 2008;8(8):4-19. https://doi. org/10.1080/15265160802317974

9. American Medical Association. AMA Code of Medical Ethics: Guidance in a Pandemic. https:// www.ama-assn.org/delivering-care/ethics/ ama-code-medical-ethics-guidance-pandemic. Published 2020. Updated Apr 14, 2020. Accessed Apr 24, 2020.

10. Boelig RC, Manuck T, Oliver EA, et al. Labor and delivery guidance for COVID-19. Am J Obstet Gynecol MFM. 2020;2(2):100110. https://doi. org/10.1016/j.ajogmf.2020.100110

11. Boelig RC, Saccone G, Bellussi F, Berghella V. MFM guidance for COVID-19. Am J Obstet Gynecol MFM. 2020;2(2):100106. https://doi. org/10.1016/j.ajogmf.2020.100106

12. Society for Maternal-Fetal Medicine and American College of Obstetricians and Gynecologists. Joint statement regarding the CDC's recent removal of pregnant people from their webpage that outlines "people who are at higher risk for severe illness” from COVID-19”. https://s3.amazonaws.com/cdn.smfm.org/media/2280/COVID CDC PPE letter.obcareproviders.pdf. Published Apr 6, 2020. Accessed Apr 22, 2020.

13. American College of Obstetricians and Gynecologists. Novel Coronavirus 2019 (COVID-19) Practice Advisory. https://www.acog.org/clinical/clinical-guidance/practice-advisory/articles/2020/03/novel-coronavirus-2019. Published Mar 2020. Updated Apr 21, 2020. Accessed Apr 21, 2020.

14. Society for Maternal-Fetal Medicine and Society for Obstetric and Anesthesia and Perinatology. Labor and Delivery COVID-19 Considerations. https://www.smfm.org/covidclinical. Published Mar 27, 2020. Updated Apr 14, 2020. Accessed Apr 21, 2020.

15. Society for Obstetric and Anesthesia and Perinatology. Interim Considerations for Obstetric Anesthesia Care Related to COVID-19. https://soap. org/education/provider-education/expert-summaries/interim-considerations-for-obstetric-anesthesia-care-related-to-covid19/. Published Mar 15, 2020. Accessed Apr 21, 2020.

16. Obstetrics and Gynecology Residency Program at the University of California SF. National Curriculum: Introducing National Remote OBGYN Resident Didactics. https://obgyn.ucsf.edu/residency-program/national-curriculum. Accessed April 24, 2020.

17. American College of Obstetricians and Gynecologists. COVID-19 FAQs for Obstetrician-Gynecologists, Obstetrics. Washington, DC: ACOG; 2020. https://www.acog.org/clinical-information/physician-faqs/covid-19-faqs-for-ob-gynsobstetrics. Accessed April 28, 2020. 